DOI: $10.1002 /$ cctc. 201402150

Highlight

\title{
Concurrent cross metathesis and enzymatic oxidation: Enabling off-equilibrium
} transformations.

\author{
Valentin Köhler*[a] and Thomas ${ }^{\wedge \wedge} \mathrm{R}$. Ward $^{[\mathrm{a}]}$ \\ [a] Department of Chemistry \\ University of Basel \\ Spitalstrasse 51, CH-4056 Basel (Switzerland) \\ Email:valentin.koehler@unibas.ch
}

H. Zhao, J.^^F. Hartwig and coworkers combined successfully homogeneous alkene metathesis and biocatalysis in a concurrent fashion. A Ru-NHC complex provides an equilibrating mixture of cross metathesis products. The selective simultaneous epoxidation by P450 BM3 enables product yields well above the hypothetical two-step process.

Enzymatic and chemocatalysis have developed widely independently to address synthetic challenges in the production of fine chemicals. The parallel development can partly be attributed to the historical division of the scientific communities from which they originate - micro- and molecular biologists on the enzymatic side, whereas organic/inorganic chemists were concerned with the development of chemocatalysis.

Both approaches provide now a wealth of methods to create added value, but can be considered complementary in many aspects, not least the range of reactions they catalyse.

Chemocatalysts have traditionally been optimized mostly for use in organic solvents. They typically accept a large substrate scope for a given transformation, but often require stringent conditions, including the exclusion of water and oxygen.

Biocatalysts in contrast, operate in complex media such as cell lysates, provide frequently excellent selectivity but often display limited substrate scope, which may be traced back to their exquisite performance. 
Combining chemo- and enzymatic catalysis in a concurrent fashion is considered challenging due to compatibility issues of the catalysts and/or the reaction conditions of the individual steps. Despite this, their combination offers opportunities to outperform sequential transformations. ${ }^{[1]}$

A successful concept where the concurrent application of chemo- and enzymatic catalysis can be exploited to particular advantage is the maintenance of an equilibrium of intermediates by means of a chemocatalyst and highly selective conversion of one of the intermediates by means of an enzymatic transformation (Scheme $\left.{ }^{\wedge \wedge} 1\right)$. This was firmly established for a wide range of dynamic kinetic resolution reactions: a secondary alcohol or an $\alpha$-branched amine is racemized by a transition metal catalyst and only one of the enantiomers is converted concurrently in a lipase-mediated acylation reaction. ${ }^{[2]}$

Zhao, Hartwig and coworkers have elegantly expanded this concept of dynamic catalyst cooperation in a proof of principle study by combining alkene metathesis, a biocomplementary reaction par excellence, with a P450-mediated alkene epoxidation. ${ }^{[3]}$

Alkene metathesis ranks amongst the most prominent organometallic catalytic reactions of the last two decades. Its popularity was boosted by the introduction of easy to handle homogeneous ruthenium and molybdenum catalysts. Although double bond geometry can now be controlled to a substantial extent in many inter- and intramolecular reactions in favor of the $Z$-isomer, cross metathesis of olefins can suffer additionally from the preferential formation of self-metathesis products. ${ }^{[4, \wedge 5]}$

P450 monooxygenases have found widespread attention as potentially versatile biocatalysts, which enable oxidation of organic substrates under mild conditions using molecular oxygen and NAD(P)H. The soluble P450 BM3 from Bacillus megaterium is particularly attractive for synthetic applications as the reductase domain is fused with the oxidative heme center in a single polypeptide chain. The enzyme has a preference for fatty acids while the catalytic efficiency depends on substrate chain length. It can give rise to both epoxidation and hydroxylation and its specificity can be substantially tuned by genetic manipulation. ${ }^{[6]}$ 
The compatibility of the transition metal-based metathesis and the enzymatic oxidation catalyst was evaluated by comparing activities of the individual players in the presence of their idling catalytic partner. For the evaluation of the monooxygenase, a saturated carboxylic acid was hydroxylated. Likewise, a suitable metathesis catalyst compatible with P450 BM3 was identified in the presence of $\mathrm{NADP}^{+}$instead of the NADPH required for the oxidation step. Both catalysts tolerated each other under the biphasic reaction conditions (aqueous/isooctane) that were selected for cooperative catalysis.

In the concurrent process, NADPH was regenerated by means of an engineered phosphite dehydrogenase (PTDH) from Pseudomonas stutzeri with substantially improved catalytic efficiency towards $\mathrm{NADP}^{+}$and higher operational stability than the wild type enzyme. This regeneration approach is less established than those employing for example, formate dehydrogenase or glucose dehydrogenase. However, it might become a true alternative for certain applications with its attractive features of high catalytic efficiency ( $\left.k_{\mathrm{cat}} / K_{\mathrm{M}, \mathrm{NADPH}}\right)$, high productivity, a low $K_{\mathrm{M}}$ value for the second substrate phosphite and good operational stability. ${ }^{[7]}$ Additionally a catalase was included to decompose $\mathrm{H}_{2} \mathrm{O}_{2}$, produced by partial uncoupling in P450 BM3. ${ }^{[6]}$

In the metathesis reaction between 10-undecenoic acid and trans-3-hexene, two products and their respective double bond isomers can be formed (other than the starting materials and not counting ethylene, Scheme ${ }^{\wedge \wedge} 2$ ). Among all alkene-containing species present in the mixture, the monooxygenase recognizes preferentially 10 -tridecenoic acid which is converted to the corresponding epoxides with moderate enantioselectivity $\left(38^{\wedge} \%\right.$ ee for the $E$-epoxide). The continual removal of 10-tridecenoic acid from the equilibrating alkene mixture by the monooxygenase leads to a higher concentration of the final product (a stereoisomeric mixture of 10,11-epoxytridecanoic acid) than would be possible in an analogous sequential transformation.

Varying the loading of 10-undecenoic acid and/or its ratio to trans-3-hexene, at constant P450 BM3 concentration but with the Ru-catalyst adjusted to $2.5^{\wedge \wedge} \mathrm{mol}^{\wedge} \%$ (relative to 10 -undecenoic acid), can lead to a substantial yield increase of up to 2.6 fold compared to the hypothetical sequential process. With $12.5^{\wedge \wedge} \mathrm{mM}$ initial loading of 10 -undecenoic acid in 
the organic phase and only 1.5 equivalents of 3 -hexene, a GC-MS yield of $70^{\wedge} \% 10,11$ epoxytridecanoic acid was obtained.

Hartwig, Zhao and coworkers have thereby demonstrated that the simultaneous application of a homogeneous olefin metathesis catalyst with a biocatalyst can be advantageous. ${ }^{[8]}$ Key to this achievement may be the compartmentalization of the homogeneous- and enzymatic catalysts in two immiscible phases. Indeed, it has been demonstrated that both catalyst classes (homogeneous catalysts and biocatalysts) can suffer from mutual inhibition. Several strategies have been successfully implemented to overcome this challenge. These include encapsulation of the organometallic catalyst within a supramolecular capsule or a host protein, phase separation of the partners by immobilisation on a solid support or running the reaction under biphasic conditions. These designs ensure that the organometallic moiety is physically separated from the enzyme thus preventing deactivation of one or both catalytic partners. ${ }^{[1]}$

The optimization of a concurrent catalytic system with two or more catalysts working in concert requires in general more effort due to additional restraints. For example the temperature maximum is dictated by the thermally least stable catalytic partner. Accordingly, it is likely that aspects of the individual steps might proceed more efficiently when they are conducted in a sequential manner. A sequential process for the current example however, would be limited in terms of yield by the equilibrium distribution of alkenes for a given set of reaction conditions.

The present system employs a substantial loading of the metathesis catalyst, which, although not at all unusual in laboratory scale metathesis reactions, may limit its large scale application due to the high cost of the ruthenium based catalyst. The development of metathesis catalysts and the engineering of reaction conditions which enable high turnover numbers in combined action with biocatalysts are accordingly of high importance. ${ }^{[9]}$

The authors extended the application of the concept in the tandem olefin crossmetathesis/epoxidation of 4-butenyloxybenzoic acid with trans-3-hexene at a yield increase of 1.3 fold compared to the hypothetical sequential process. Other applications may be imagined such as the selective processing of one double bond isomer from an equilibrating mixture of 
intermediates if the respective enzymatic partner for the desired subsequent transformation can be identified. Enzymatic partners are in the first instance limited to biocatalysts which transform double bonds (directly or indirectly) so that the products are not subject to alkene metathesis. Apart from the required specificity for the choice intermediate, compatibility with the metathesis conditions needs to be given or achieved. The technologies to tailor biocatalysts to specific needs also brighten the Future for potential simultaneous chemoenzymatic cascades. ${ }^{[10]}$

$<$ lit1>C.^^A. Denard, J.^^F. Hartwig, H. Zhao, ACS Catal. 2013, 3, 2856--2864.

$<$ lit2>I. Hussain, J.-E. Bäckvall in Enzyme Catalysis in Organic Synthesis, 3rd^ed. (Eds.: K. Drauz, H. Gröger, O. May), Wiley-VCH, Weinheim, 2011, pp.^^1777--1806.

$<$ lit3>C. ${ }^{\wedge}$ A. Denard, H. Huang, M.^^J. Bartlett, L. Lu, Y. Tan, H. Zhao, J.^^F. Hartwig, Angew. Chem. 2014, 126, 475--479; Angew. Chem. Int. Ed. 2014, 53, 465--469.

$<$ lit4>A. Fürstner, Science 2013, 341, 1357.

$<$ lit5>S.^^J. Connon, S. Blechert, Angew. Chem. 2003, 115, 1944--1968; Angew. Chem. Int. Ed. 2003, 42, 1900--1923.

$<$ lit6>C.^^J.^^C. Whitehouse, S.^^G. Bell, L.-L. Wong, Chem. Soc. Rev. 2012, 41, 1218-1260.

$<$ lit7>T.^^W. Johannes, R.^^D. Woodyer, H. Zhao, Biotechnol. Bioeng. 2007, 96, 18--26.

$<$ lit8>K. Tenbrink, M. Seßler, J. Schatz, H. Gröger, Adv. Synth. Catal. 2011, 353, 2363-2367.

$<$ lit9>K. Grela, Ł. Gułajski, K. Skowerski in Metal-Catalyzed Reactions in Water (Eds.: P. ${ }^{\wedge}$ H. Dixneuf, V. Cadierno), Wiley-VCH, Weinheim, 2013, pp.^^291--336.

$<$ lit10>U.^^.$^{\wedge}$. Bornscheuer, G. ${ }^{\wedge}$ W. Huisman, R. ${ }^{\wedge}$ J. Kazlauskas, S. Lutz, J. ${ }^{\wedge}$ C. Moore, K. Robins, Nature 2012, 485, 185--194.

Received: March 20, 2014

Revised: April 7, 2014 


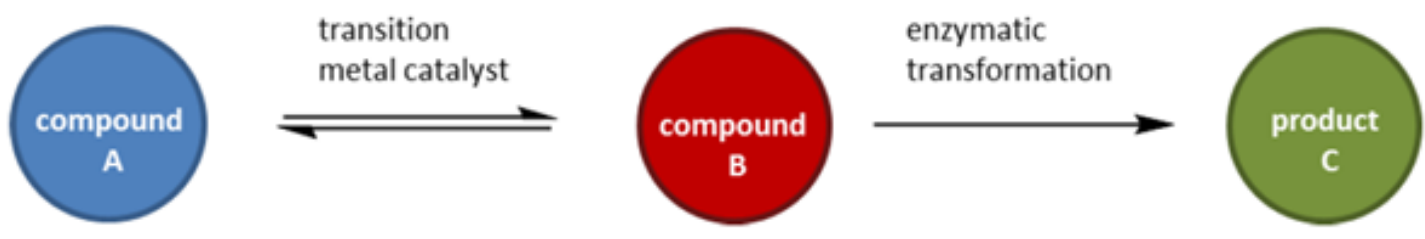

Scheme ${ }^{\wedge \wedge} 1$ A successful concept in concurrent chemo-enzymatic catalysis combines a reversible transition metal catalyzed reaction with an irreversible and highly specific

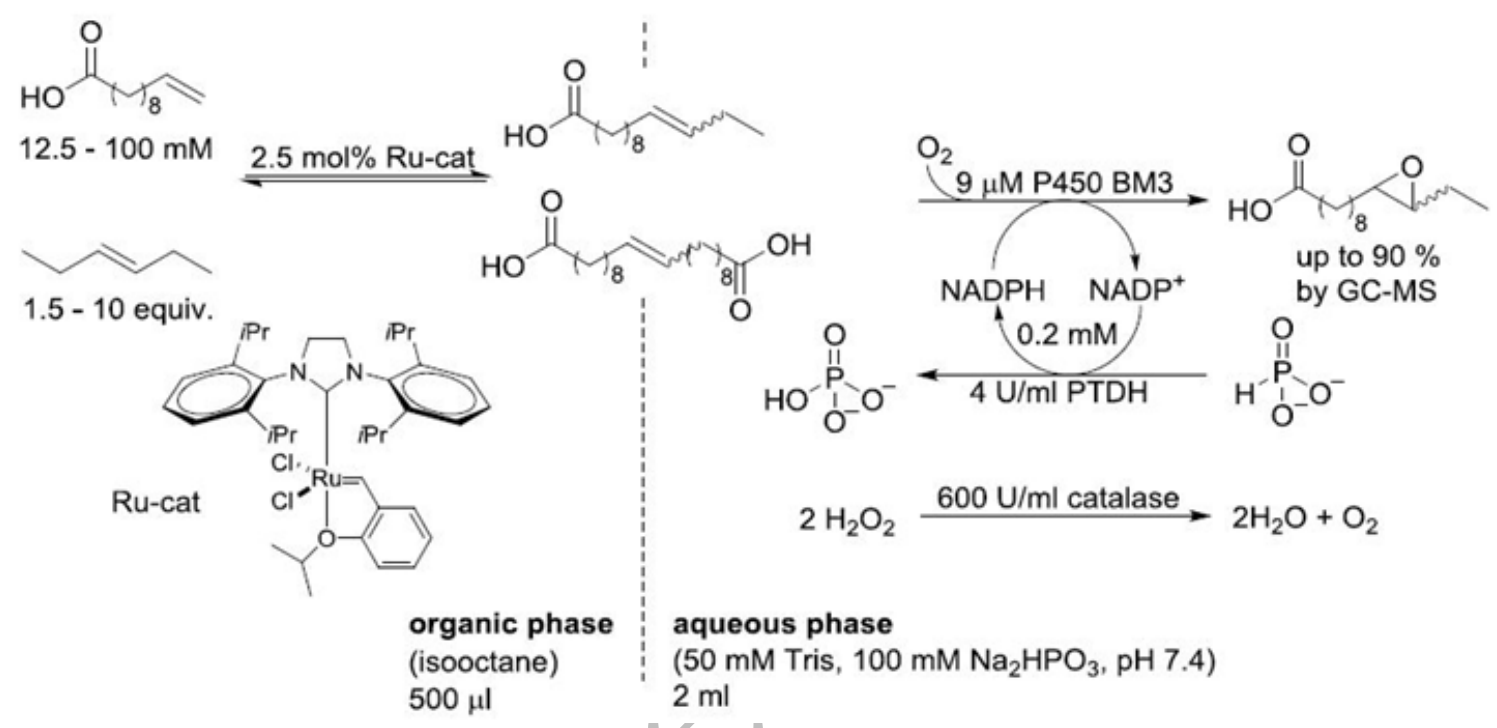

Scheme ${ }^{\wedge} 2$ Biphasic reaction Scheme for the cooperative conversion of 10-undecenoic acid and trans-3-hexene by a Ru-based metathesis catalyst and P450 BM3. Phosphite dehydrogenase (PTDH) is employed for NADPH regeneration. Catalase prevents the accumulation of $\mathrm{H}_{2} \mathrm{O}_{2}$ caused by uncoupling in the $\mathrm{P} 450 \mathrm{BM} 3$ monooxygenase. $E / Z=6: 1$ for the epoxide product. Adapted from reference ${ }^{\wedge \wedge} 3$ with permission from Wiley. 